\title{
Treatment Response in Depressed Patients with Enhanced Ca Mobilization Stimulated by Serotonin
}

\author{
Ichiro Kusumi, M.D., Ph.D., Katsuji Suzuki, M.D., Yuki Sasaki, B.S., Kensuke Kameda, M.D., Ph.D., \\ and Tsukasa Koyama, M.D., Ph.D.
}

Serotonin (5-HT)-stimulated intraplatelet calcium ( $\mathrm{Ca}$ ) mobilization has been shown to be enhanced in nonmedicated depressive patients by many studies. However, there has not been any longitudinal follow-up study of this parameter. We examined the relationship between treatment response and pretreatment value of the 5-HT-induced Ca response. The 5-HT(10 $\mu M)$-induced intraplatelet Ca mobilization was measured in 98 nonmedicated depressive patients (24 bipolar disorders, 51 melancholic major depressive disorders, and 23 nonmelancholic major depressive disorders). These patients were followed up prospectively for a further period of five years. The depressed patients with enhanced Ca response to 5-HT in bipolar disorders exhibited a good response to mood stabilizers but those with major depressive disorders showed a poor response to antidepressants. These findings suggest the possibility that the 5-HT-induced intraplatelet Ca response may be a good predictor of treatment response in depressed patients. Longer longitudinal follow-up studies are needed in larger samples to examine if this parameter may be a specific biological marker for unipolar-bipolar dichotomy. [Neuropsychopharmacology 23:690-696, 2000] ( 2000 American College of Neuropsychopharmacology. Published by Elsevier Science Inc.
KEY WORDS: Calcium; Serotonin; Mood disorder; Treatment response; Platelet

A large number of studies have indicated that central serotonin-2A $\left(5-\mathrm{HT}_{2 \mathrm{~A}}\right)$ receptor dysfunction is an important factor in the etiology of mood disorders. Increased densities of 5- $\mathrm{HT}_{2 \mathrm{~A}}$ receptor binding sites in the postmortem brain of depressed patients or suicide victims, and platelets of depressed patients have been reported by many investigators (see review: Kusumi and Koyama 1998). An neuroendocrine study also suggested the supersensitivity of $5-\mathrm{HT}_{2 \mathrm{~A}}$ receptors by showing that cortisol response to oral D, L-5-hydroxy-

From the Department of Psychiatry, Hokkaido University School of Medicine, Sapporo, Japan.

Address correspondence to: Ichiro Kusumi, M.D., Ph.D., Department of Psychiatry, Hokkaido University School of Medicine, North 15, West 7, Sapporo 060-8638, Japan. 2000. tryptophan was enhanced in nonmedicated depressed and manic patients (Meltzer et al. 1984). By measuring 5-HT-induced intraplatelet calcium (Ca) mobilization, we have already indicated that $5-\mathrm{HT}_{2 \mathrm{~A}}$ receptor function is enhanced in nonmedicated patients with bipolar disorders and melancholic major depression compared to normal controls (Kusumi et al. 1991b, 1994).

Although these findings were confirmed by several studies (Mikuni et al. 1992; Eckert et al. 1993; Okamoto et al. 1994; Konopka et al. 1996; Tomiyoshi et al. 1999), there has not been any follow-up study of this parameter except our previous report (Kusumi et al. 1994), which suggested that the enhanced Ca response is not state dependent, but trait dependent. Assuming that this parameter might be some predictor of treatment response in depressed patients, in this study, we examined the relationship between clinical improvement and pretreatment value of the 5-HT-induced intraplatelet Ca response in patients with bipolar disorders and major depressive disorders. 


\section{SUBJECTS AND METHODS}

\section{Subjects}

The study reports on a total of 98 drug-free, major depressive patients and 30 normal controls. After complete description of the study, written informed consent was obtained from all participants. The depressed patients were diagnosed and subtyped using DSM-IV criteria, which consisted of 24 bipolar disorders, 51 major depressive disorders with melancholic feature, and 23 major depressive disorders without melancholic feature. For at least four weeks before blood sampling, they had not received any psychotropic medication or any other drugs such as aspirin that might interfere with platelet aggregation. They were all free of physical illness including hypertension. The severity of depressive symptoms was assessed by the 17-item Hamilton Rating Scale for Depression (HRSD) at the time of sampling.

Family history was investigated whether or not there was any patient with mood disorders within a first- or second-degree relative. The clinical data for subgroups of nonmedicated patients and normal controls are shown in Table 1.

\section{Treatment Response}

All patients were observed by the psychiatrists to check the responsiveness to the treatment at least monthly for a period of five years after blood sampling. Medications at follow-up were prescribed based on clinical judgment rather than by random assignment to a particular medication protocol. Responders were defined as patients with absence of DSM-IV (hypo)manic or major depressive episodes during at least two years of effective treatment despite a reasonably high risk of recurrence (history of at least one affective episode during the one year preceding the current episode) for bipolar disorders, and as a reduction to five or less of total HRSD score for major depressive disorders.

On the other hand, nonresponders were patients who experienced at least one affective episode for a period of less than two years with onset after trials of various combination with adequate dose of mood stabilizers for bipolar disorders, and those who did not respond to an adequate period (more than four weeks) of treatment with at least two different antidepressants at optimal dosage (a minimum of the equivalent of 150 $\mathrm{mg}$ imipramine per day) for major depressive disorders.

\section{5-HT-Stimulated Ca Response}

The isolation of platelets and the measurement of intraplatelet Ca concentration were performed as described previously (Kusumi et al. 1991a, 1994). Briefly, platelet- rich plasma was incubated with $4 \mu \mathrm{M}$ fura-2/acetoxymethylester, a Ca sensitive fluorescent probe, for 15 $\min$ at $37^{\circ} \mathrm{C}$. After centrifugation, the resulting platelet pellet was suspended at $1 \times 10^{8}$ cells $/ \mathrm{ml}$ in KrebsRinger HEPES buffer. The samples were prewarmed in a cuvette at $37^{\circ} \mathrm{C}$ for $4 \mathrm{~min}$ and then $10 \mu \mathrm{M}$ 5-HT was added to the incubation medium. Fluorescence was measured on a Hitachi F-2000 fluorometer with excitation at 340 and $380 \mathrm{~nm}$, and with emission at $510 \mathrm{~nm}$. Intracellular $\mathrm{Ca}$ concentrations were calculated from the ratio of fluorescence intensities at two excitation wavelengths in the platelet samples according to the method of Grynkiewicz et al. (1985).

We examined both resting $\mathrm{Ca}$ concentration and maximum Ca response $(\%$ increase $=$ initial peak $(\mathrm{nM}) /$ resting level $(\mathrm{nM}) \times 100)$ induced by $10 \mu \mathrm{M}$ of $5-\mathrm{HT}$. Since data expressed by percent increase is more stable (lower inter- or intra-assay) than those by $\mathrm{Ca}$ increase (initial peak minus resting Ca level) as suggested in the previous report (Kusumi et al. 1991a), we analysed data with percent increase in this longitudinal follow-up study.

\section{Statistical Analysis}

Results are expressed as means \pm SD. Statistical analysis was performed using Student's t-test, Mann-Whittney's U test, or one-way analysis of variance (ANOVA) for multiple comparison followed by Scheffe's test. Analysis by $\chi^{2}$ was used to assess the relationship between pretreatment $\mathrm{Ca}$ mobilization and clinical response. $P$-values less than .05 were considered statistically significant.

\section{RESULTS}

\section{Clinical Characteristics of Depressive Patients}

Comparing the demographic data for subgroups of nonmedicated depressive patients, there were no significant differences in sex ratio, age, family history, or total HRSD score among three groups (Table 1). The maximal Ca response induced by $10 \mu \mathrm{M}$ of $5-\mathrm{HT}$ was significantly higher in patients with bipolar disorders than in normal controls, whereas no significant difference in basal Ca concentration was observed among four groups (Table 1).

We did not find any difference in both basal Ca level and 5-HT-stimulated Ca response between male and female when examining for total or each subgroup of depressed patients (data not shown). Thus, further analyses were performed with male and female patients together. No significant correlation was observed between total HRSD score and the 5-HT-induced Ca mobilization in the entire group or each subgroup of patients (data not shown). 
Table 1. Clinical Data for Subgroups of Nonmedicated Depressive Patients and Normal Controls

\begin{tabular}{|c|c|c|c|c|}
\hline & $\begin{array}{c}\text { Bipolar } \\
\text { disorders }\end{array}$ & $\begin{array}{c}\text { Major depressive } \\
\text { disorder } \\
\text { with melancholic } \\
\text { features }\end{array}$ & $\begin{array}{c}\text { Major depressive } \\
\text { disorder } \\
\text { without melancholic } \\
\text { features }\end{array}$ & Controls \\
\hline $\mathrm{N}$ & 24 & 51 & 23 & 30 \\
\hline Male/Female & $10 / 14$ & $21 / 30$ & $12 / 11$ & $14 / 16$ \\
\hline Age (year) & $36.8 \pm 14.6$ & $42.1 \pm 16.3$ & $39.1 \pm 15.2$ & $42.8 \pm 11.0$ \\
\hline Family history $^{a}$ & $4(17 \%)$ & $9(18 \%)$ & $5(22 \%)$ & \\
\hline $\mathrm{HRSD}^{b}$ & $21.3 \pm 6.1$ & $23.4 \pm 7.6$ & $20.3 \pm 5.6$ & \\
\hline Resting Ca level (nM) & $68.5 \pm 17.3$ & $69.1 \pm 15.9$ & $69.9 \pm 20.9$ & $76.2 \pm 13.1$ \\
\hline 5-HT-induced Ca response (\%) & $272.1 \pm 58.3^{* *}$ & $247.6 \pm 50.5$ & $221.9 \pm 32.7$ & $225.5 \pm 27.8$ \\
\hline
\end{tabular}

${ }^{a}$ Family history of mood disorders in a first- or second degree relative.

${ }^{b}$ Hamilton rating scale for depression.

${ }^{* *} p<.005$ vs. controls.

\section{Treatment Response in Depressed Patients}

First, we examined clinical characteristics and the 5-HTstimulated $\mathrm{Ca}$ mobilization for responder and nonresponder in our present sample. In bipolar disorders, although there were no significant differences in age, family history, or total HRSD score between responder and nonresponder, the 5-HT-induced Ca mobilization in responder was significantly higher than in nonresponder (Table 2). Basal Ca level in responder was significantly lower than in nonresponder.

On the other hand, in melancholic major depressive disorder, the 5-HT-stimulated Ca mobilization in responder was significantly lower than in nonresponder (Table 3). No significant differences were observed in age, family history, total HRSD score, or basal Ca concentration between both groups (Table 3). In nonmelancholic major depressive disorder, there were no significant differences in the 5-HT-induced Ca response, basal Ca level, or other clinical index between responder and nonresponder (Table 4).

\section{Characteristics of Depressive Patients with Enhanced Ca Response}

To examine the role of increased Ca response, we classified the patients as "enhanced" and "the others" groups according to their pretreatment value of the 5-HTinduced $\mathrm{Ca}$ response. The cut-off value chosen was $280 \%$ of basal, since it corresponded to the value of the mean + 2SD for normal controls (Table 1 ). The number of patients with a Ca response higher than this value was $24(24.5 \%$ of total), of which $10(40 \%)$ were bipolar disorders, 13 (26\%) were melancholic major depressive disorders, and only one (4\%) was a non-melancholic major depressive disorder (Table 5). The demographic characteristics of nonmedicated depressive patients with a Ca response above $280 \%$ and below $280 \%$ are shown in Table 5 . There were no significant differences in age, family history or total HRSD score between the two groups. Basal Ca level in patients with a Ca response above $280 \%$ was significantly lower than in those below $280 \%$ (Table 5 ).

Next, we examined the treatment response for patients with a pretreatment Ca response above $280 \%$ and below $280 \%$. The clinical response to mood stabilizers in the patients with bipolar disorders are shown in Table 6. Chi-squared analysis of the data revealed a significantly higher response rate in patients with a pretreatment Ca response above $280 \%$ than those below $280 \%$ $(60 \%$ vs. $7 \%, p=.005, N=24)$. Final effective treatments were all lithium carbonate, of which three were co-administered with sodium valproate. There was no difference in medical approach between responders

Table 2. Response to Mood Stabilizers in Patients with Bipolar Disorders

\begin{tabular}{lccc}
\hline & Responder & Nonresponder & t-test \\
\hline $\mathrm{N}$ & 7 & 17 & \\
Male/Female & $2 / 5$ & $8 / 9$ & N.S. \\
Age (year) & $40.9 \pm 18.4$ & $35.2 \pm 12.9$ & N.S. \\
Family history $^{a}$ & $1(14 \%)$ & $3(18 \%)$ & N.S. \\
HRSD $^{b}$ & $23.8 \pm 3.7$ & $20.5 \pm 6.6$ & N.S. \\
Resting Ca level (nM) $_{\text {5-HT-induced Ca response (\%) }}$ & $56.8 \pm 13.2$ & $73.3 \pm 16.8$ & $p<.05$ \\
& $331.4 \pm 51.2$ & $247.8 \pm 41.7$ & $p<.0005$ \\
\hline
\end{tabular}

${ }^{a}$ Family history of mood disorders in a first- or second degree relative.

${ }^{b}$ Hamilton rating scale for depression.

N.S., not significant. 
Table 3. Response to Antidepressants in Patients with Melancholic Major Depressive Disorder

\begin{tabular}{lccc}
\hline & Responder & Nonresponder & t-test \\
\hline $\mathrm{N}$ & 25 & 26 & \\
Male/Female & $11 / 14$ & $10 / 16$ & N.S. \\
Age (year) & $41.0 \pm 16.3$ & $43.1 \pm 16.6$ & N.S. \\
Family history & $4(16 \%)$ & $5(19 \%)$ & N.S. \\
HRSD $^{b}$ & $23.5 \pm 7.5$ & $23.3 \pm 7.8$ & N.S. \\
Resting Ca level (nM) $_{\text {5-HT-induced Ca response (\%) }}$ & $69.3 \pm 16.4$ & $68.9 \pm 15.8$ & N.S. $^{c}$ \\
\hline
\end{tabular}

${ }^{a}$ Family history of mood disorders in a first- or second degree relative.

${ }^{b}$ Hamilton rating scale for depression.

'Mann-Whittney's U test.

N.S., not significant.

and nonresponders because all patients were treated with one of mood stabilizers (firstly lithium carbonate, and secondly sodium valproate or carbamazepine), then in case of no efficacy, the various combinations of these agents. The clinical response to antidepressants in the patients with major depressive disorders are also shown in Table 6.

In contrast with the result for bipolar disorders, patients with a Ca response above $280 \%$ showed a significantly lower response rate than those below $280 \%(14 \%$ vs. $58 \%, p .=.003$ for total patients, $N=74 ; 15 \%$ vs. $61 \%$, $p=.005$ for melancholic major depressive disorder, $N=$ $51 ; 0 \%$ vs. $55 \%, p=.48$ for non-melancholic major depressive disorder, Fisher's exact method, $N=23$ ). $5-\mathrm{HT}_{2 \mathrm{~A}}$ receptor antagonists, amoxapine $(46 \%$ of responder) or setiptiline (11\%) appeared to be effective for major depressive patients with a Ca response below $280 \%$ (Table 6). There was no difference in medical approach between responders and nonresponders because most patients were firstly treated with amoxapine or clomipramine, secondly imipramine, sulpiride, or tetracyclic antidepressants, then in case of no efficacy, bromocriptine or novel selective 5-HT, and/or noradrenaline reuptake inhibitor.

\section{DISCUSSION}

The present results indicate that depressed patients with enhanced Ca response to 5-HT in bipolar disorders exhibit a good response to mood stabilizers but that those in major depressive disorders show a poor response to antidepressants. These findings suggest the possibility that the 5-HT-induced intraplatelet Ca mobilization may be a good predictor of treatment response in depressed patients. Many investigators have confirmed our previous finding that the 5-HT-induced Ca response is enhanced in some types of mood disorders as mentioned in the introduction, but there has not been any longitudinal follow-up study of this parameter except our previous report (Kusumi et al. 1994), which showed that the enhanced Ca response to 5-HT is not normalized after the remission of depressive symptoms. This is the first follow-up study examining the relationship between treatment response and pretreatment 5-HT-induced Ca mobilization in both patients with bipolar disorders and major depressive disorders.

As shown in our previous report (Kusumi et al. 1991a), the 5-HT-stimulated intraplatelet Ca mobilization is a stable marker since it is not significantly af-

Table 4. Response to Antidepressants in Patients with Nonmelancholic Major Depressive Disorder

\begin{tabular}{lccc}
\hline & Responder & Nonresponder & t-test \\
\hline $\mathrm{N}$ & 12 & 11 & \\
Male/Female & $7 / 5$ & $5 / 6$ & N.S. \\
Age (year) & $36.1 \pm 15.6$ & $42.5 \pm 14.9$ & N.S. \\
Family history $^{a}$ & $4(33 \%)$ & $1(9 \%)$ & N.S. \\
HRSD $^{b}$ & $21.8 \pm 4.6$ & $18.6 \pm 6.2$ & N.S. \\
Resting Ca level (nM) $_{\text {5-HT-induced Ca response (\%) }}^{73.3 \pm 16.7}$ & $66.2 \pm 24.9$ & N.S. \\
\hline
\end{tabular}

${ }^{a}$ Family history of mood disorders in a first- or second degree relative.

${ }^{b}$ Hamilton rating scale for depression.

N.S., not significant. 
Table 5. Characteristics of Nonmedicated Depressive Patients with Enhanced Ca Response

\begin{tabular}{lccc}
\hline & $\begin{array}{c}\text { Ca response } \\
>\mathbf{2 8 0} \%\end{array}$ & $\begin{array}{c}\text { Ca response } \\
<\mathbf{2 8 0} \%\end{array}$ & t-test \\
\hline $\mathrm{N}$ & 24 & 74 & \\
Male/Female & $12 / 12$ & $31 / 43$ & N.S. \\
Age (year) $^{a}$ & $42.3 \pm 16.2$ & $39.4 \pm 15.5$ & N.S. \\
Family history $^{a}$ & $5(21 \%)$ & $13(18 \%)$ & N.S. \\
RRSD $^{b}$ & $23.7 \pm 5.7$ & $21.6 \pm 7.1$ & N.S. \\
5-HT-induced Ca response (\%) & $60.8 \pm 17.6$ & $71.2 \pm 17.1$ & $p<.01$ \\
Bipolar disorder & $318.0 \pm 35.3$ & $224.8 \pm 31.6$ & \\
Melancholic major depressive disorder $_{\text {Nonmelancholic major depressive disorder }}$ & $10(40 \%)$ & $14(60 \%)$ & \\
\hline
\end{tabular}

${ }^{a}$ Family history of mood disorders in a first- or second degree relative.

${ }^{b}$ Hamilton rating scale for depression.

N.S., not significant.

fected by sampling time and season, sex, age, meal, or exercise in healthy subjects. This study showed that sex and age did not significantly influence this parameter in depressed patient sample. The 5-HT-induced Ca mobilization in responder was significantly different from that in nonresponder for patients with bipolar disorders and melancholic major depressive disorder, although there were no significant differences in clinical characteristics such as the severity of depressive symptoms and the family history. In bipolar disorders, responder to mood stabilizers exhibited a significant higher $\mathrm{Ca}$ response to 5-HT than nonresponder, whereas responder to antidepressants showed a significant lower Ca response than nonresponder in melancholic major depressive disorder. In nonmelancholic major depressive disorder, no significant difference was found in the 5-HT-stimulated Ca mobilization between responder and nonresponder. Moreover, com- paring enhanced $\mathrm{Ca}$ response group which showed more than $280 \%$ of basal with the other one, similar results were obtained for both bipolar disorders and melancholic major depressive disorder. In bipolar disorders, the group with enhanced $\mathrm{Ca}$ mobilization demonstrated a significantly higher response to mood stabilizers than the other one. In melancholic major depressive disorder, however, the former exhibited a significantly lower response to antidepressants than the latter.

These findings indirectly suggest that bipolar disorder and major depressive disorder may be due to the different pathophysiology even if a part of patients in both disorders show enhanced Ca response to 5-HT. In fact, ratio of patients with enhanced $\mathrm{Ca}$ response was different among the three subgroups of depressive patients. Bipolar disorder was most frequent $(40 \%)$, followed by melancholic major depressive disorder (26\%), and nonmelancholic major depressive disorder $(4 \%)$.

Table 6. Ratio of Patients with a Good Treatment Response for Bipolar Disorder and Major Depressive Disorders

\begin{tabular}{|c|c|c|c|}
\hline Patient group & $N$ & $\begin{array}{c}\text { Good } \\
\text { treatment } \\
\text { response }\end{array}$ & Effective treatments \\
\hline \multicolumn{4}{|l|}{ Bipolar disorder } \\
\hline Ca response $>280 \%$ & 10 & $6(60 \%)$ & $\mathrm{Li} 4, \mathrm{Li} / \mathrm{VPA} 2$ \\
\hline Ca response $<280 \%$ & 14 & $1(7 \%)$ & $\mathrm{Li} / \mathrm{VPA} 1$ \\
\hline \multicolumn{4}{|c|}{ Major depressive disorder with melancholic features } \\
\hline Ca response $>280 \%$ & 13 & $2(15 \%)$ & BRM 2 \\
\hline Ca response $<280 \%$ & 38 & $23(61 \%)$ & $\begin{array}{l}\text { AMX 11, AMX/BRM 2, IMI 3, CMI } 2 \\
\text { IMI/TRZ 1, TRZ 1, MNP 1, MCI 1, DLX } 1\end{array}$ \\
\hline \multicolumn{4}{|c|}{ Major depressive disorder without melancholic feature } \\
\hline Ca response $>280 \%$ & 1 & $0(0 \%)$ & \\
\hline Ca response $<280 \%$ & 22 & $12(55 \%)$ & $\begin{array}{l}\text { STP 4, AMX 3, MPL } 2 \text { CMI 1, MIA 1, } \\
\text { SUL } 1\end{array}$ \\
\hline
\end{tabular}

Abbreviations: AMX, amoxapine; BRM, bromocriptine; CMI, clomipramine; DLX, duloxetine; IMI, imipramine; Li, lithium carbonate; MCI, MCI-225 (selective noradrenaline reuptake inhibitor); MIA, mianserin; MNP, milnacipran; MPL, maprotiline; STP, setiptiline; SUL, sulpiride; TRZ, trazodone; VPA, sodium valproate. 
Moreover, in contrast with our previous report (Kusumi et al. 1994), in the present study using a larger sample, the 5-HT-stimulated Ca response in melancholic major depressive disorder was not significantly different from that in normal control (Table 1). Therefore, it is possible that the enhanced Ca response may be a specific marker for bipolar disorders. In the previous study, a group of melancholic major depression could include potential bipolar patients, which might influence the results. Indeed, during the follow-up observation, the diagnosis was changed from major depressive disorder to bipolar disorder in two patients with enhanced Ca response. In this sense, the diagnosis of monopolar depression may be changeable, thus longer longitudinal follow-up study is necessary in a larger sample in future.

It is interesting to note the final effective treatments for responder in our sample. In bipolar disorders, all responders exhibited a good response to lithium carbonate. On the other hand, in melancholic major depressive disorder, 13 of 23 responders (57\%) in the patients with a pretreatment Ca response below $280 \%$ showed a good response to $5-\mathrm{HT}_{2 \mathrm{~A}}$ receptor antagonist amoxapine. In nonmelancholic major depressive disorder, seven of 12 responders $(58 \%)$ among the patients with a $\mathrm{Ca}$ response below $280 \%$ indicated a good response to $5-\mathrm{HT}_{2 \mathrm{~A}}$ receptor antagonist setiptiline or amoxapine. We expected that patients with an enhanced Ca response to 5-HT exhibited a good response to 5- $\mathrm{HT}_{2 \mathrm{~A}}$ receptor antagonist, thus this result is completely reverse. There may be several possible reasons for this unexpected finding. First, the cut-off value for the enhanced Ca response $(280 \%)$ was so high that clinical dose of $5-\mathrm{HT}_{2 \mathrm{~A}}$ receptor antagonist failed to inhibit the 5-HT-induced Ca response. However, this possibility is not plausible because inhibition of Ca mobilization is not necessarily correlated with treatment response in our preliminary study. Second, the enhanced Ca response observed in depressed patients may not be specifically mediated by $5-\mathrm{HT}_{2 \mathrm{~A}}$ receptor. It has been previously reported that thrombin-stimulated $\mathrm{Ca}$ response was enhanced in nonmedicated depressive patients with bipolar disorders compared to those with major depression and normal control (Dubovsky et al. 1991; Kusumi et al. 1992). In bipolar disorders, there might be some alterations in intracellular signal transduction that are common to both 5-HT- and thrombin-induced Ca mobilizing pathways. This possibility is partly supported by the reports that hyperfunction of GTP binding protein is observed in the leukocytes of bipolar patients (Schreiber et al. 1991; Young et al. 1994). Agonist-stimulated Ca response in neutrophils from patients with bipolar disorders was also enhanced, which was significantly attenuated by lithium treatment (Foerstner et al. 1994). These findings may suggest that bipolar disorders are associated with a nonspecific hypersensitivity of the inositol phospholipid second-messenger generating system. As mentioned above, if enhanced Ca response might be a specific marker for bipolar disorders, it does not necessarily conflict with the results that the patients with enhanced Ca mobilization exhibited a good response to mood stabilizers and a poor response to antidepressants including $5-\mathrm{HT}_{2 \mathrm{~A}}$ receptor antagonists.

As for the basal Ca concentration, most researchers, except Dubovsky et al. (1991) and Konopka et al. (1996), have shown that there is no significant difference between depressed patients and normal controls (Kusumi et al. 1991b, 1994; Mikuni et al. 1992; Eckert et al. 1993; Okamoto et al. 1994; Bothwell et al. 1994; Tomoyoshi et al. 1999). In this study, this finding was reconfirmed when comparing by DSM-IV subtype (Table 1). However, responders in bipolar disorder had lower resting Ca level than nonresponders (Table 2), although no significant differences were observed in major depressive disorder with and without melancholic features (Tables 3 and 4). Moreover, basal Ca level in patients with a Ca response above $280 \%$ was significantly lower than in those below $280 \%$ (Table 5).

It is not plausible that higher 5-HT-induced Ca response may be due to lower basal Ca concentration since the same findings were observed when examining by absolute Ca increase (initial peak minus resting Ca level) instead of percent increase. The reason for lower basal Ca level in bipolar responder is unknown from the present study. However, it is not reasonable to assume that the basal Ca concentration can predict the $\mathrm{Ca}$ responsiveness to $5-\mathrm{HT}$ because there was no significant correlation between the resting $\mathrm{Ca}$ level and 5-HT-induced Ca increase in patients with bipolar disorder $(N=24, \mathrm{r}=0.31, p=.13$, Pearson's correlation coefficient). Considering the report of Tan et al. (1990) that the basal Ca concentration was higher in lithium-treated euthymic bipolar patients than in controls, the possibility exists that effective lithium treatment may elevate basal Ca level by affecting the mechanism for maintaining intracellular Ca level such as $\mathrm{Ca}$ pump, Ca storing, and forming complexes of Ca with intracellular proteins and phospholipids. Therefore, it seems to be a good possibility that low resting Ca level may predict a good responsiveness to mood stabilizers for bipolar disorder, which should be reconfirmed in further studies.

In conclusion, the depressed patients with enhanced Ca mobilization exhibited a good response to mood stabilizers but those in major depressive disorders show a poor response to antidepressants. These findings suggest the possibility that the 5-HT-induced intraplatelet Ca response may be a good predictor of treatment response in depressed patients. In future, longer longitudinal follow-up studies are needed in larger samples to examine that this parameter may be a specific biological marker for unipolar-bipolar dichotomy. 


\section{ACKNOWLEDGMENTS}

This work was partly supported by grants from the Akiyama Foundation, the Japanese Research Foundation for Clinical Pharmacology, and the Pharmacopsychiatry Research Foundation.

\section{REFERENCES}

Dubovsky SL, Lee C, Christiano J, Murphy J (1991): Elevated platelet intracellular calcium concentration in bipolar depression. Biol Psychiatry 29:441-450

Eckert A, Riemann GD, Aldenhoff J, Mueller WE (1993): Elevated intracellular calcium levels after $5-\mathrm{HT}_{2}$ receptor stimulation in platelets of depressed patients. Biol Psychiatry 34:565-568

Foerstner U, Bohus M, Gebicke-Haerter PJ, Baumer B, Berger M, van Calker D (1994): Decreased agonist-stimulated $\mathrm{Ca}^{2+}$ response in neutrophils from patients under chronic lithium therapy. Eur Arch Psychiatry Clin Neurosci 243:240-243

Grynkiewicz G, Poenie M, Tsien RY (1985): A new generation of $\mathrm{Ca}^{2+}$ indicators with greatly improved fluorescence properties. J Biol Chem 260:3440-3450

Konopka LM, Cooper R, Crayton JW (1996): Serotonininduced increases in platelet cytosolic calcium concentration in depressed, schizophrenic, and substance abuse patients. Biol Psychiatry 39:708-713

Kusumi I, Koyama T, Yamashita I (1991a): Effect of various factors on serotonin-induced $\mathrm{Ca}^{2+}$ response in human platelets. Life Sci 48:2405-2412

Kusumi I, Koyama T, Yamashita I (1991b): Serotonin-stimulated $\mathrm{Ca}^{2+}$ response is increased in the platelets of depressed patients. Biol Psychiatry 30:310-312

Kusumi I, Koyama T, Yamashita I (1992): Thrombin-induced platelet calcium mobilization is enhanced in bipolar disorder. Biol Psychiatry 32:731-734

Kusumi I, Koyama T, Yamashita I (1994): Serotonin-induced platelet intracellular calcium mobilization in depressed patients. Psychopharmacology 113:322-327

Kusumi I, Koyama T (1998): Serotonin-2A receptor function in affective disorders. In Ozawa $\mathrm{H}$, Saito $\mathrm{T}$, Takahata $\mathrm{N}$ (eds), Signal Transduction in Affective Disorders. Tokyo, Japan: Springer, pp 21-34

Meltzer HY, Umberkoman-Wiita B, Robertson A, Tricou BJ, Lowy M, Perline R (1984): Effect of 5-hydroxytryptophan on serum cortisol levels in major affective disorders. I. Enhanced response in depression and mania. Arch Gen Psychiatry 41:366-374

Mikuni M, Kagaya A, Takahashi K, Meltzer HY (1992): Serotonin but not norepinephrine-induced calcium mobilization of platelets is enhanced in affective disorders. Psychopharmacology 106:311-314

Okamoto Y, Kagaya A, Shinno H, Motohashi N, Yamawaki S (1994): Serotonin-induced platelet calcium mobilization is enhanced in mania. Life Sci 56:327-332

Schreiber G, Avissar S, Danon A, Belmaker RH (1991): Hyperfunctional $\mathrm{G}$ proteins in mononuclear leukocytes of patients with mania. Biol Psychiatry 29:273-280

Tan CH, Javors MA, Seleshi E, Lowrimore PA, Bowden CL (1990): Effects of lithium on platelet ionic intracellular calcium concentration in patients with bipolar (manicdepressive) disorder and healthy controls. Life Sci 46:1175-1180

Tomiyoshi R, Kamei K, Muraoka S, Muneoka K, Takigawa M (1999): Serotonin-induced platelet intracellular $\mathrm{Ca}^{2+}$ responses in untreated depressed patients and imipramine responders in remission. Biol Psychiatry 45:1042-1048

Young LT, Li PP, Kamble A, Siu KP, Warsh JJ (1994): Mononuclear leukocyte levels of $\mathrm{G}$ proteins with bipolar disorder or major depressive disorder. Am J Psychiatry 151:594-596 